\title{
Prótesis implanto soportada sobre barra en maxilar reconstruido por injerto de peroné
}

Implant-supported prosthesis on it sweeps in reconstruction maxillary for fibula graft.

\section{Resumen}

El desarrollo de técnicas microvasculares permite cl uso de injertos óseos libres vascularizados representando un imporlante avance en el tratamiento de defectos maxilares.

Las ventajas del peroné es que ofrece un abundante reemplazo del hueso bicortical para la reconstrucción de defectos segmentarios atraviesan la línea media mandibular, csto permite la posibilidad de insertar impantes dentales. El peroné es considerado como el mejor sitio donador para un largo periodo de reconstrucción y ofrece varias ventajas comparadas con la cresta iliaca, omoplato y radio.

La oseointegracion a revolucionado la rehabilitación dental, en adición el uso de la tomografía computarizada dimensional y biomodelos para hacer mas preciso el planeamiento y ejecucion de los casos del complejo cráneo raxilofacial. Debido al alto éxito a largo plazo (índice de restauraciones proiésicas con implantes mas del $90 \%$ ) implantes inmediatos o diferidos. Esta técnica puede ser considerada en reconstrucción maxilofacial.

Prucedimientos adicionales al hueso injertado incluyendo la distracción vertical del peroné injertado son usados para lograr una adecuada función y resultados estéticos

\footnotetext{
Abstract

The development of microvascular techniques enabling the use of free vascularized bone grafts represents an important advance in the treatment of maxillary defects.

The advantages of the fibula are that they offer an abundant supply of tubed bicortical bone for reconstruction of segment defects across the midline, it allows the possibility of subsequent insertion of dental implants.

The fibula is considered as the best donor site for long-span reconstruction and offers several advantages compared with ilium, scapula, and radius.

The ossointegration has revolutionized the dental rehabilitalion, in addition the use of three dimensional computed tomography and biomodels had made the planning and execution of complex craniomaxillofacial cases more precise. Due to the high long term succes (rate of prosthetics restorations with implants more than $90 \%$ ) inmediate or delayed implants. This technic should be considerer in maxillofacial reconstruction,

Additional bone grafting procedures, including the vertical distraction of the grafted fibula are used to achieve adequate function and aesthethics results.
}

\section{Introducción}

La reconstrucción maxilar y la rehabilitación bucal es uno de los más grandes desafíos afrontados por los cirujanos buco maxilofaciales

Algunos procedimientos convencionales usando injertos óseos o implantes aloplasticos pueden inducir a complicaciones semejantes como reabsor-ción del injerto óseo o exposición del implante. Los defectos óseos en el área de la cirugía cráneo maxilofacial son bastantes comunes. Esto ocurre en situaciones post-traumáticas graves o des-

\section{Artículo Clínicos}

\section{Jorge Omar Huamani Mamani'; José Luis Cornejo Salazar²*; Karen Manrique Ponce De Leún'}

\footnotetext{
Consulta privada, Av. San Luis 1700 San Borja Lima, Perú.

2 Departamento Académico de Estomatología Médico Quirúrgico

* Facultad de Odontología, Universidad Nacional Mayor de San Marcos, Lima, Perú E-mail: jcornejos@unusm.edu.pe
}

Palabras clave: reconstrucción maxilar, Injerto de peroné, Implante dental, Prótesis implanto soportada

Key words: maxillary recorstruction, Fibula free flap, Dental implants, Implant-supported prosthesis

pués de procedimientos oncológicos de cabeza y cuello. Presentamos un paciente con gran defecto cráneo maxilofacial reconstruido con injerto libre de peroné y posterior rehabilitación prutésica. Este caso se realizo con el equipo de cirujanos maxilofaciales que lidera el Profescr Ferri durante el entrenamiento en el Servicio de Estomatología y Cirugía MaxiloFacial del Hospital Roger Salengro de Francia. Se presenta porque es inédito en nuestro medio $y$ es fundamental el conceplo de trabajo en equipo multi e interdisciplinario.

\section{Caso clínico}

Paciente masculino de 31 años que fue hospitalizado en el Servicio de Emergencias por tentativa de suicidio con arma de fuego. Se trataba de un traumatismo cráneo-maxilofacial por bala con orificio de entrada submentoniana.

Al examen inicial se observa deterioro cutáneo medio facial con una fractura múltiple de la mandíbula, un orificio de salida a nivel de las fosas nasales y del etmoides (Figs 1, 2). Existe igualmente pérdida cutánea a 
nivel de la glabela y de la bóveda frontal con fractura y salida de contenido cerebral.

El examen tomografico del macizo facial observamos múltiples trazos de fractura que comprometen el piso anterior del macizo etmoidal, las paredes orbitarias internas y superiores con defecto óseo importante en la región parasinfisiaria. Sobre el plano facial se observan lesiones medio faciales, se estableció una vía aérea mediante traqueotomía y reducción maxilar con fijación externa.

La fractura del piso anterior del cráneo ha necesitado una intervención neuroquirurgica con injertu iliaco; la reconstrucción de la premaxila por un injerto de peroné (fig. 4) microanastomosado tomado a nivel de la pierna izquierda.

El paciente ha estado presentando algunos dolores de la pierna izquierda. Lo que sc ha controlado con analgesicos opiodes, En el mismo tiempo operatorio se realiza un injerto libre a nivel del dorso nasal asi mismo una reconstrucción orbitaria con coralina (xenoinjerto).

En un segundo tiempo son colocados los implantes en la diafisis del peroné (Figs.5,6).

Posteriormente una barra colada permite la realización de una prótesis implanto soportada sobre ella despuès de decolar el colgajo cutáneo. (Figs. 7-12).

\section{Resultados}

La reconstrucción microvascular para un gran defecto de la cabeza y del cuello es un método seguro, produciendo excelentes resultados con tasas de $92 \%-98 \%$ y es señalado como un método moderno estándar para defectos extensos en esta región.

Sin embargo según estos mismos estudios anatómicos el colgajo vascularizado del peronć ${ }^{1,23}$, es apto para la oseointegracion de los implantes dentales a largo plazo, por la buena estructura bicortical dado por la gran interfase hueso-implante ${ }^{1}$

La estabilidad primaria es importante en los implantes colocados en los injertos ello permitirá una restauración segura y previsible ${ }^{5}$.

Esto requiere un planeamiento y un equipo multidisciplinario, incluyendo cirujanos con experiencia microquirurgica y prostodonticas.

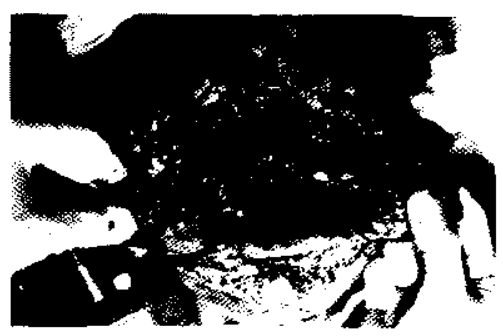

Fig. 1: Emergencia A

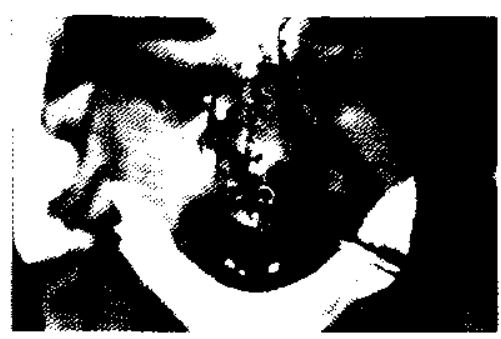

Fig.3: Cirugía en emergencia

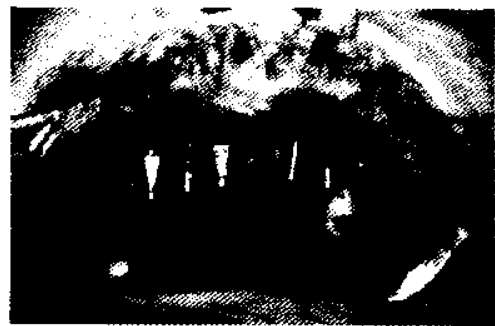

Fig. 5: Instalación de los implantes (pines)

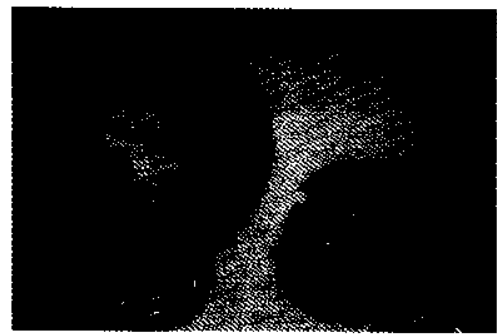

Fig. 7: Barra y prótesis (laboratorio)

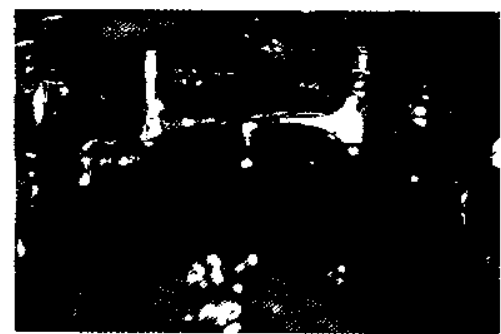

Fig. 9: Barra sobre implantes A (izq.)

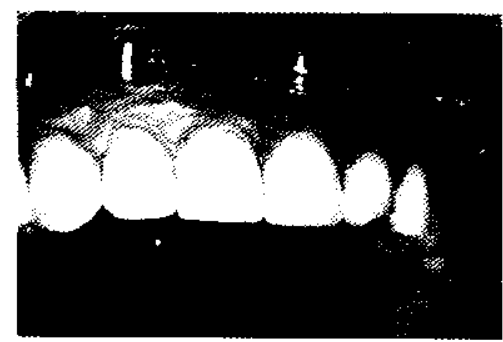

Fig. 11: Prótesis instalada retenida sobre la barra

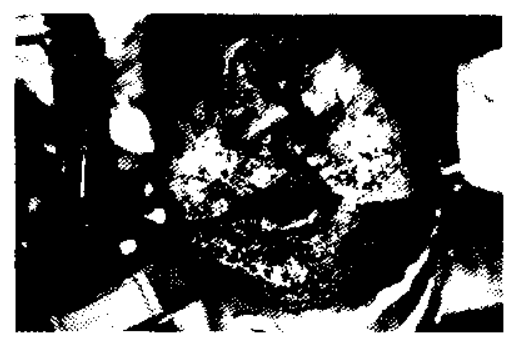

Fig.2: Emergencia B

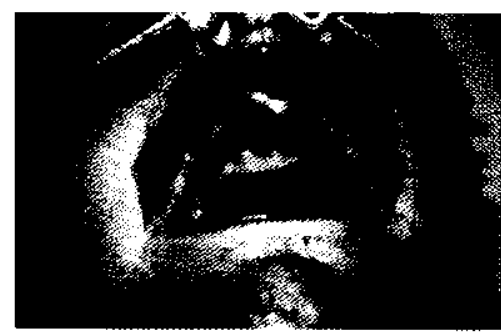

Fig.4: Injerto de peroné en maxilar

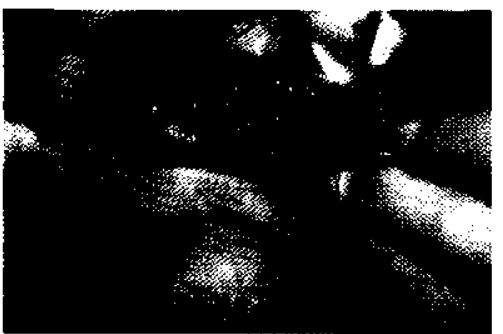

Fig. 6: Instalación de los implantes (tornillos de cicatrización)

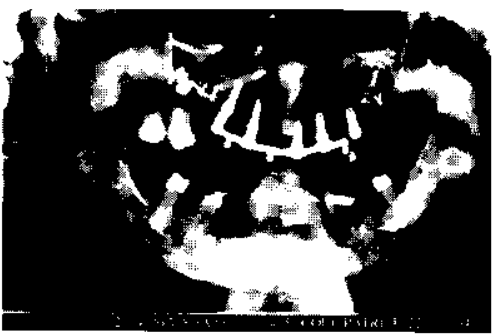

Fig. 8: Radiografía Panorámica

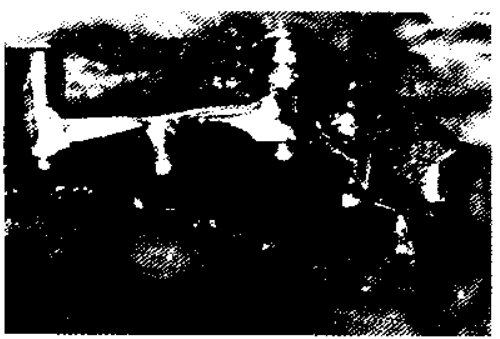

Fig. 10: Barra sobre implantes A (der.)

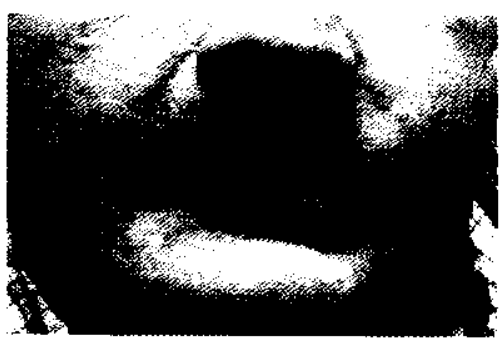

Fig. 12: Paciente con la prótesis instalada 
Las secuelas encontradas son minimas, el colgajo libre de peroné es nues tra primera elección de colgajos óseos ${ }^{6}$.

\section{Discuslón}

Los objetivos de la reconstrucción maxilar deben de recaer sobre 3 pilares 1) para proveer soporte salisfactorio y permitir una rehabilitación protésica, 2) para restaurar el lenguaje, 3) para mejorar la apariencia facial'.

El cambio fundamental en reconstrucción de la cabeza y del cuello en los últimos 20 años a sido la introducción de la transferencia de tejido libre vascularizado.

Taylor (1975) ${ }^{8}$, Hidalgo (1989) 9 fue el primero en usar el injerto libre vascularizado de perone en la reconstrucción para mandibula y varias técnicas avanzadas fucron desarrolladas subsecuentemente.

Nakayama y Cols $(1995)^{10}$ lo emplearon en la reconstrucción maxilar

La creación de anastomosis scguras de la cabeza es la más importante precondición para una transferencia exitosa?.

El uso de los colgajos vascularizados óseos ofrece ventajas, transplantando células de hueso vivo debido a la restauración de la irrigación vascular.

La reabsorción es mínima especialmente comparada con injertos de hueso no vascularizado.

El colgajo libre de peroné es el más comúnmente utilizado de los colgajos libres en reconstrucción mandibular conteniendo hueso vascularizado ${ }^{6}$.

Combinando la reconstrucción del hueso vascularizado con prótesis implantosoportadas, fijas/removibles (sobredentaduras) adicionan una significativa calidad a la reconstrucción.

El « complejo funcional maxilar» a sido reconocido por muchos años.Este complejo funcional puede ser comparado con una sistema completo hecho de estructuras de barras y soportes. Esta estructura es el resultado de car- gas forzadas impuestas en el hueso.

Es evidente que son 2 las reglas para la reconstrucción biomecánica del maxilar:

Primero, la reconstrucción podría ser hecha con una estructura ligera, compuesta de un hueso alveolar en herradura soportando fuerzas y favoreciendo la masticación recargando la base del cráneo?.

Este objetivo es logrado con el colgajo libre de peroné, que lo puede reconstruir sin riesgo.

En casos de una sustancial perdida de tejido, es esencial usar un injerto vascularizado, para proveer hueso vivo capaz de responder una estimulación mecánica eludiendo una reabsorción inicial.

Segundo, adicionando fuerzas al hueso es necesario para evitar una reabsorción a largo plazo. Este requerimiento enfatiza las ventajas de las prótesis implantosoportadas ${ }^{7}$.

El tratamiento optimiza las relaciones maxilares, aumentando cl resultado estético de la reconstrucción ofreciendo una continuidad ósea, la lijación rigida del colgajo en el hueso receptor puede ser hecho con mini placas o micro placas ${ }^{5}$.

\section{Agradecimiento}

Al Profesor Doctor Joel Ferri MD PhD Medico estomatólogo, Jefe del Servicio de Estomatología y Cirugía Maxilofacial del Hospital Roger Salengro de Lille, Irancia por su calidad humana y su generosidad docente-asistencial

\section{Referencias}

1.A. Picard, J Tiguemounine, A. Chabut, D. Goga. Reconstruction d'une perte de substance complexe du maxillaire par transfert de fibula vascularisé après réalisation d'une fistule arlério-veinteuse par boucle vasculaire saphène, Revue de Stomatologie et de Chirurgic Maxillofaciale, novembre 2004, Volume 105 No 5 .

2.Dennis Rohner, Peter Bucher, Christoph Kunz, Beat Hammer,
Joachim Prein, Robert $K$. Schenk. Treatment of severe atrophy of the maxilla with the prefabricated free vascularized fibula flap, Journal Clinical Oral Implants Research13, $2002 ; 44-52$

3.De Santis, G. M.D.; Nocini, P. F M.D., D.D.S.; Chiarini, L. M.D., D.D.S.; Bedogni, A. M.D. Functional Rehabilitation of the Atrophic Mandible and Maxilla with Fibula Flaps and Implant-Supported Prosthesis. Plastic \& Reconstructive Surgery. 113(1):88-98, January 2004.

4.Franz-Josef Kramer, Rupert Dempf, Bernd Bremer. Efficacy of dental implants placed into fibula-free flaps for orofacial reconstruction, JournalClinical Oral Implants Research 16, $2005 ; 80-88$

5.Claude Jaquiéry, Dennis Rohner, Christoph Kunz, Peter Bucher, Françoise Peters, Robert K. Schenk. Reconstruction of maxillary and mandibular defects using prefabricated microvascular fibular grafts and osseointegrated dental implants a prospective study, JournalClinical Oral Implants Research 15, 2004 ; 598-606

6.J. Braga-Silva, M.R.O. Jacger and P.P.S. Favalli. Reconstruction mandibulaire: les lambeaux microchirurgicaux de crète iliaque et péroné, Annales de Chirurgie Plastique Esthétique, Volume 50, Issue 1, February 2005, Pages 49-55.

7.Joel Ferri, François Caprioli, Guy Peuvrel and Jean-Marie Langlois. Use of the fibula free flap in maxillary reconstruction: A report of 3 cases, Journal of Oral and Maxillofacial Surgery, Volume 60, Issue 5, May 2002, Pages 567-574

8. Taylor Gl, Miller GD, Ham FJ. The free vascularized bone graft. A clinical extension of microvascular techniques. Plast Reconstr Surg. 1975 May;55 (5):533-41.

9. Hidalgo DA. Fibula free flap: a new method of mandible reconstruction. Plast Reconstr Surg. 1989 Jul; $84(1): 71-9$

10. Nakayama B, Matsuura $H$, Ishihara O, Hasegawa H, Mataga I, Torii S. Functional reconstruction of a bilateral maxillectomy defect using a fibula osteocutaneous flap with osseointegrated implants. Plast Reconstr Surg. 1995 Oct; 96(5):1201-4. 